\title{
Performance Evaluation of the Dynamic Trajectory Design for an Unmanned Aerial Base Station in a Single Frequency Network
}

\author{
Margot Deruyck*, Alberto Marri ${ }^{\dagger}$, Silvia Mignardi ${ }^{\dagger}$, Luc Martens* Wout Joseph* and Roberto Verdone $^{\dagger}$ \\ *Department of Information Technology, Ghent University - IMEC - WAVES, Ghent, Belgium, \\ email: margot.deruyck@ugent.be \\ ${ }^{\dagger}$ DEI, University of Bologna, Bologna, Italy
}

\begin{abstract}
Using an Unmanned Aerial Base Station (UABS) i.e., a base station carried by a UAV (Unmanned Aerial Vehicle) or drone, is a promising approach to offer coverage and capacity to those users that are not being served by the base stations of the terrestrial network. In this paper, we propose an approach to the design of the drone's trajectory to account for the quickly varying user traffic and pattern. This approach is based on the identification of clusters made of nearby users to be served. The decision on which cluster to visit next by the UABS depends on a cost-function considering the distance to the next cluster, the user density and spread in the cluster, and the direction compared to the previously visited cluster. Furthermore, we propose a radio resource assignment algorithm to minimize the interference from the UABS to the terrestrial network when both are operating in the same frequency band. The potential improvements in terms of network capacity (sum throughput) and user satisfaction are estimated in this study.
\end{abstract}

\section{INTRODUCTION}

The behaviour of mobile data users has tremendously changed in the last decade. The next generation standard i.e., the 5th Generation (5G), for mobile radio communications, which is currently under development, should not only be supporting significant higher data rates, but also offer different services to mobile users and platforms, with heterogeneous requirements. Furthermore, the $5 \mathrm{G}$ network will be serving a massive density of nodes both human-held and machine-type. This tendency can already be noticed today with the emerging Internet of Things (IoT). Due to this large density of wireless nodes, we also need to revise the design of the wireless network. Currently, network planning is based on average or even peak traffic predictions. However, the estimated increase of node density implies a larger deviation in the traffic generation process than today. Using the same design will only result in largely suboptimal solutions.

The future wireless networks will be characterized by a high degree of flexibility compared to the past. This flexibility will allow them to adapt smoothly, autonomously and efficiently to the quickly changing traffic demand evolutions both in time and in space. Unfortunately, this will not be possible if the infrastructure of these networks will remain static, as it is in current deployments: the offered capacity on a local scale is highly dependent on the density of the infrastructure equipment in the considered area. Mobile infrastructure nodes can provide a solution to this issue.

In this paper, a network supported by Unmanned Aerial Vehicles (UAVs), also known as drones, is considered. An Unmanned Aerial Base Station (UABS), which is a base station mounted on a UAV, flies over the network consisting of various Terrestrial Base Stations (TBSs), serving the traffic that can not adequately be covered by the terrestrial network. To serve the unsatisfied users, the UABS will fly along a certain trajectory defined in cooperation with the network. The backhaul connection of the UABS to the network can be provided through high capacity Line-of-Sight (LoS) wireless backhaul links (e.g., by using millimeter-wave or visible light communication), but is beyond the scope of this study. In this paper, we focus on the dynamic design of a single UABS's trajectory and the issue of interference from the UABS to the terrestrial network while delivering high throughput video services to the unsatisfied users. The trajectory is based on the identification of clusters made of nearby users to be served as proposed by [10]. The novelty of our trajectory design is the fact that it is eventdriven, meaning that once a UABS arrives in a certain cluster, we are able to define its next target cluster onthe-fly. This allows us to respond to the very dynamically and quickly changing user traffic in future wireless networks. Furthermore, to the best of our knowledge, an interference avoidance approach from the UABS to the terrestrial network when operating in the same frequency band has not been proposed before. Both approaches are applied on a urban environment where users move and request video downloads with a minimal throughput.

The outline of the paper is as follows. Section II describes the state-of-the-art literature on UAV aided networks. In Section III, we propose the scenario, our 
dynamic trajectory design, and interference avoidance approach. Section IV discusses the performance of the overall network when applying the approaches of Section III. Finally, we summarize our conclusions in Section V.

\section{RELATION TO THE LITERATURE}

Early works about base stations mounted on UAVs analyze the propagation model affecting the Air-toGround (ATG) channel of drone terminals. Line of Sight (LoS) probability and the elevation angle between the UABS and the ground node are found to have the major role [1], [2], [3].

The work in [4] considers multiple UAVs having the aim to maintain an overall network connectivity in a 3D scenario with several ground nodes; optimal positioning of drones goes through particle swarm optimization, minimizing the number of links and costs. Furthermore, clusterization algorithms for UABSs placement and trajectories are introduced in [5], [6]; the former has the purpose to achieve the minimum energy consumption with exactly one UAV per cluster in an uplink scenario, and the latter focuses on finding a single point with 2D coordinates (height fixed) for the UAV-mounted access point to serve the highest possible number of users.

Interference issues are analyzed and modelled in [7]. The interferers are other UABSs, each transmitting towards a ground node; however, the presence of the mobile network is not considered.

In [9], UAVs were used as relays. The model uses density and cost functions to calculate areas with higher demands and multiple UAVs are deployed depending on these functions. The work in [8] investigates if drone mounted femtocell base stations can be a solution to provide coverage in a disaster scenario, when the existing infrastructure is no longer available.

The authors' aim is to introduce the concept of dynamic trajectory discovery in a mobile network scenario with TBSs and thousands of ground nodes that can potentially remain unsatisfied dependently on the network behaviour. Our model is different from previous works for the dynamic trajectory discovery, the continuous availability of service while flying and the approach of handling interference. In fact, the impact of the path and system performance are analysed for the case of a single carrier usage for both TBSs and the UABS.

\section{Methodology and mathematical model}

\section{A. Scenario}

As reference scenario, we consider a square area with side $L$ and spacing between the sites $S$, as proposed in [10] and shown in Fig. 1. A squared layout is assumed for the base station sites. Each base station site consists of four TBSs, each having a quadrisectorial directional antenna. Furthermore, $N_{S C}$ Small Cells (SCs) are uniformly and randomly distributed over the area. Table I summarizes the most important parameters related to the mobile network. The values of Table I are assumed unless mentioned otherwise. As starting point for the UABS, we considered the center of the area as shown in Fig. 1.

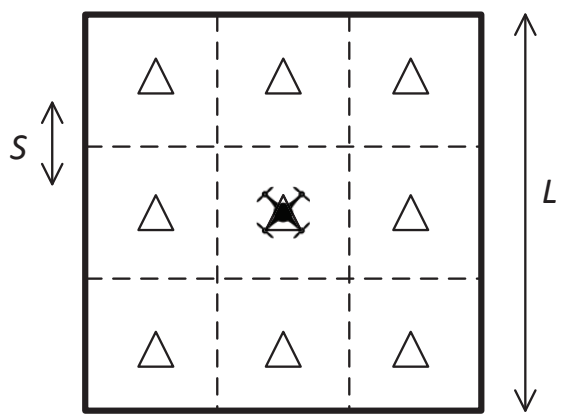

Fig. 1. Considered scenario.

TABLE I

PARAMETER VALUES FOR THE MOBILE NETWORK.

\begin{tabular}{|l|c|c|}
\hline Parameter & Symbol & Value \\
\hline Side squared area & $L$ & $6000 \mathrm{~m}$ \\
\hline Spacing between sites & $S$ & $2000 \mathrm{~m}$ \\
\hline Number of SCs & $N_{S C}$ & 50 \\
\hline Transmit power TBS & $P_{T x, T B S}$ & $43 \mathrm{dBm}$ \\
\hline Transmit power SC & $P_{T x, S C}$ & $33 \mathrm{dBm}$ \\
\hline Transmit power UABS & $P_{T x, U A B S}$ & $0 \mathrm{dBm}$ \\
\hline Transmit gain TBS & $G_{T x, T B S}$ & $12 \mathrm{dBi}$ \\
\hline Transmit gain SC & $G_{T x, S C}$ & $0 \mathrm{dBi}$ \\
\hline Transmit gain UABS & $G_{T x, U A B S}$ & $0 \mathrm{dBi}$ \\
\hline Receiver gain UE & $G_{R x}$ & $12 \mathrm{dBi}$ \\
\hline Noise figure UE & $F$ & $10 \mathrm{~dB}$ \\
\hline Coverage range SC & $r_{S C}$ & $100 \mathrm{~m}$ \\
\hline Carrier frequency TBS & $f_{T B S}$ & $800 \mathrm{MHz}$ \\
\hline Carrier frequency SC & $f_{S C}$ & $10 \mathrm{GHw}$ \\
\hline Carrier frequency UABS & $f_{U A B S}$ & $800 \mathrm{MHz}$ \\
\hline Height UABS & $h_{U A B S}$ & $50 \mathrm{~m}$ \\
\hline Bit rate per subcarrier & $R_{b, m n}$ & $30 \mathrm{kbps}$ \\
\hline
\end{tabular}

The users arrive in the area according to a Poisson process with arrival rate $\lambda_{u}$ per second per $\mathrm{km}^{2}$. The users can either move or not during a simulation. The movement of the users is simulated through a Random Way Point model with time stamps of $20 \mathrm{~s}$ as proposed in [10]. The users are requesting to download a video through a streaming service, requiring a minimum throughput, and accept a maximum delay to start downloads. All parameters related to the user traffic are shown in Table II. As propagation model, we consider the one proposed in [2], [3].

\section{B. Design of the dynamic trajectory}

The trajectory of the UABS is based on the clustering of the unsatisfied users. The UABS will fly from centroid to centroid of these clusters. The clustering algorithm 
TABLE II

PARAMETERS USER TRAFFIC.

\begin{tabular}{|l|c|}
\hline Parameter & Value \\
\hline Arrival requests per second $\lambda_{u}$ & 10 arrivals/s \\
\hline Minimum throughput requested & $10 \mathrm{Mbps}$ \\
\hline Traffic demand per UE & $25 \mathrm{MB}$ \\
\hline Maximum accepted waiting time & $24 \mathrm{~s}$ \\
\hline$S N R_{\min }$ & $10 \mathrm{~dB}$ \\
\hline$S I R_{\min }$ & $3 \mathrm{~dB}$ \\
\hline
\end{tabular}

for the unsatisfied users is the hierarchical centroid linkage [11]. However, user traffic is very dynamical and defining the complete path of the UABS at the start might not be very useful. People might already have left the area or withdrawn their request when the UABS arrives at their cluster. Therefore, in this paper, we propose to define the UABS's trajectory on-the-go. Once the UABS arrives at a centroid, the clustering algorithm is again applied and we decide which cluster to visit next. For each of the clusters, a visiting cost is calculated. The next cluster is then the cluster with the lowest value for this cost. The cost $C_{i}$ for cluster $i$ is determined as follows:

$$
C_{i}=\left(\frac{d_{i}}{d_{t h}(K)}+W(K) \cdot \frac{\delta}{\delta_{\max }}\right) \cdot(1+A(L))
$$

The first fraction in Eq. (1) i.e., $\frac{d_{i}}{d_{t h}(K)}$, represents the fly distance from the current position to the cluster centroid, while the second fraction i.e., $\frac{\delta}{\delta_{\max }}$, represents the user density within the cluster. The different parameters and functions are defined as follows:

- $K$ : the cluster cardinality,

- $d_{i}$ : the distance between the UABS's current position and the centroid of cluster $i$ as shown in Fig. 2,

- $d_{t h}(K)$ : a threshold distance depending on $K$. Whenever the fly distance is larger than the threshold distance i.e., $d_{i}>d_{t h}$, the corresponding cluster is not eligible as next cluster. The more clusters $K$ we have, the lower this threshold will be. The function is obtained through spline interpolation from:

$$
d_{t h}(K) \begin{cases}2500 & K=10 \\ 2000 & K=20 \\ 1500 & K=50 \\ 1000 & K=100\end{cases}
$$

For $K>100, d_{t h}(K)=1000$. When decreasing the cluster cardinality, the mean distance among the clusters and the drone increases. The threshold distance has to follow this behaviour to avoid the discard of a high number of clusters and letting the parameter distance become the main one.

- $W(K)$ : a function depending on $K$ to give the same weight to the fly distance as to the user density. It is obtained through spline interpolation from:

$$
W(K) \begin{cases}1 & K=100 \\ 1.18 & K=500 \\ 1.27 & K=1000\end{cases}
$$

$W(K)$ is considered to be 1 for $K<100$. When increasing the cluster cardinality, some clusters consists of only a few nodes, resulting in a high density and low value of delta $a_{i} /$ delta $_{T h}$. For this reason, the density component of the cost function turns into the leading one, and the drone would fly over a zone with few nodes. To avoid this situation, this component of the cost function is multiplied by $W(K)$, which is higher when the cluster cardinality is higher. In this way, the same weight for the two cost functions' components is obtained.

- $\delta$ : the mean distance inside the $i$-th cluster between the UEs and the centroid (with $\delta>0$ ). If $\delta=0$, the cluster is composed only by one node, and is for this reason discarded.

- $\delta \max :=\max \left(\delta_{1}, \delta_{2}, \ldots, \delta n\right), \delta_{n}$ being the distance between the $n$-th UE and the centroid of its cluster as shown in Fig. 2,

- $L$ : half of the length of the size of the square with as center the current position of the UABS as shown in Fig. 2,

- $A(L)$ : a function accounting for the past direction as a function of $L$. If the centroid of cluster $i$ lays in the square with size $2 \cdot L$ around the current position of the UABS, an extra cost is added to avoid that the UABS is moving back and forward between two positions. This additional cost depends on how close the cluster $i$ is located compared to the current position of the UABS.

The functions $d_{t h}(K), W(K)$ and $A(L)$ and their arbitrary values are studied and designed in order to give the same weight to each component of the cost function and to ensure a minimum spatial fairness. To obtain them, several simulations were performed: from the Cumulative Distribution Function of the different elements in the cost function and the resulting trajectory of the drone. The functions are obtained heuristically.

\section{Avoiding interference from UABS to the terrestrial network}

So far, the fact that the UABS introduces interference to the terrestrial network when operating in the same frequency band has been neglected. In this section, we propose an approach to avoid this kind of interference. This approach is based on the Radio Resource Unit (RU) assignment to the users. First, the TBSs and SCs assign resources. Since the access to the network is based on an OFDMA (Orthogonal Frequency Division Multiple Access), the minimum amount of RUs that can be assigned to the user corresponds to a Physical Resource Block (PRB), which is composed of 12 subcarriers [10]. 




Fig. 2. Identifying the different parameters for the cost-function to determine the next cluster to visit. The triangles represent the unsatisfied users.

The TBSs and SCs supply resources by considering the link conditions and applying scheduling techniques. These techniques try to assign each RU available to get a maximum exploitation of resources. Table III shows the assumptions made related to the Radio Resource Management. To assign the RUs, a slightly modified Round Robin and Proportional Fair scheduling algorithm are applied successively. [10] The first aims at allocating resources up to the minimum requested; the latter is utilized when there are still RUs available after the Round Robin scheduling to enhance the perceived quality.

TABLE III

AIR INTERfaCe PARAMETERS [10]

\begin{tabular}{|l|c|}
\hline Parameter & Value \\
\hline Subcarrier spacing & $15 \mathrm{kHz}$ \\
\hline Number of subcarriers per PRB & 12 \\
\hline Maximum capacity $C_{\max }$ & $100 \mathrm{Mbps}$ \\
\hline Time slot interval & $0.5 \mathrm{~ms}$ \\
\hline Frame time duration & $10 \mathrm{~ms}$ \\
\hline Bandwidth of TBS & $20 \mathrm{MHz}$ \\
\hline Bandwidth of SC & $1.74 \mathrm{MHz}$ \\
\hline Reuse factor & 1 \\
\hline
\end{tabular}

Next, the UABS assigns resources. Since both the terrestial network and the UABS are operating in the same frequency band, they both have access to the same pool of RUs. Therefore, to avoid interference with the terrestrial network, we limit the pool of RUs available for the UABS. To this end, the footprint of the UABS is determined as shown in Fig. 3. The footprint of the UABS is here defined as a cone with as center the UABS, a height of $h_{f l y}$ (i.e, the fly height of the UABS), and a radius of $h_{f l y} \cdot \cot \frac{\pi}{12}$. An angle of $\frac{\pi}{12}$ corresponds with $15^{\circ}$ which is the maximum angle under which we have coverage according to the ATG propagation model proposed in [2], [3]. Lower evaluation angles have a limited probability of receiving any signal from the UAV [3]. Furthermore, the selected simulation setup of [3] can only produce results for down to about $15^{\circ}$ only. The RUs that are assigned from the terrestrial network to the users present within the footprint of the UABS are then excluded from the UABS's pool of radio resources as shown in Fig. 3. The UABS can now start assigning RUs to the unsatisfied users it should cover by using the same Round Robin and Proportional Fair scheduling algorithms as mentioned above.

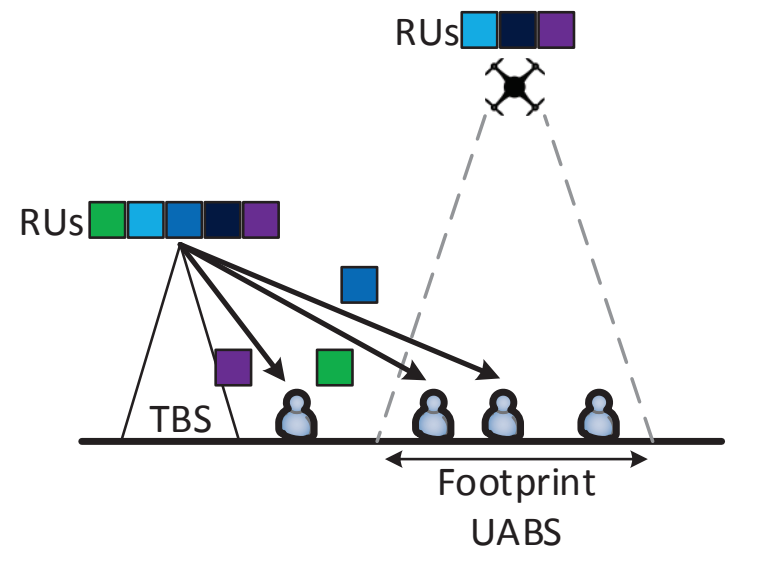

Fig. 3. Radio resource assignment to avoid interference from the UABS to the terrestrial network.

\section{Metrics}

To assess the performance of the proposed algorithms and approaches, the following metrics are evaluated: the sum throughput, the user outage rate, and the number of used RUs (when accounting for interference). The sum throughput $S T h$ (in bps) for a certain timestamp $t_{i}$ is the sum of throughputs perceived by all links (TBS-UE, SC (Small Cell)-UE and UABS-UE) in the network [10]:

$$
S T h\left(t_{i}\right)=\sum_{m=1}^{M} T h_{m}\left(t_{i}\right)
$$

with $M$ the number of users active in the network, and $T h_{m}\left(t_{i}\right)$ the throughput (in bps) achieved at UE $m$ on the instant $t_{i}$. The latter is determined as follows [10]:

$$
T h_{m}\left(t_{i}\right)=\frac{B}{N} \sum_{n=1}^{N} c_{m, n} \log _{2}\left(1+X_{m, n}\right)
$$

with $B$ the total bandwidth (in MHz), $N$ the total number of subcarriers, $c_{m, n}$ equals 1 or 0 depending on whether 
subcarrier $n$ is assigned to UE $m$ or not. $X_{m, n}$ is determined by the following equation ${ }^{1}[10]$ :

$$
\begin{aligned}
X_{m, n} & =\min \left(S N R_{m, n}, S I R_{m, n}\right) \\
S I R_{m, n} & =\frac{P_{r,\{m, n\}}}{\sum_{i=1}^{N_{M B S} P_{r,\{i, n\}}}} \\
S N R_{m, n} & =\frac{P_{r,\{m, n\}}}{2 \cdot N_{0} \cdot B_{\text {subc }}}
\end{aligned}
$$

$P_{r,\{m, n\}}$ represents the useful power received at UE $m$ on subcarrier $n, N_{M B S}$ is the number of interferers, $N_{0}$ the bilateral noise density depending on the noise figure, and $B_{\text {subc }}$ the equivalent bandwidth to obtain the noise power. The mean sum throughput determined over all timestamps is here considered.

The user outage rate $R_{\text {out }}$ is the ratio between the number of UEs $N_{\text {out }}$ remaining unserved and the total number of UEs $N_{t o t}$ present:

$$
R_{\text {out }}=\frac{N_{\text {out }}}{N_{\text {tot }}}
$$

Each of the performance metrics described above are evaluated by varying some key parameters such as the UABS's speed, its height or transmit power. Furthermore, we are also interested in the improvement in these metrics compared to the scenario without using an UABS.

\section{NUMERICAL RESUlTS}

\section{A. Introduction of the dynamic trajectory design}

In this section, we investigate the performance of our dynamic trajectory design. Fig. 4 shows the improvement (percentage) of the sum throughput compared to the scenario without a UABS for varying UAV speed and different cluster cardinalities.

Introducing a UABS in the network combined with our proposed dynamic trajectory design has a positive influence on the network performance. Depending on the considered speed and cluster cardinality, the sum throughput increases between $0.8 \%$ to almost $1.6 \%$ (sum throughput without UABS equals $65.9 \mathrm{Gbps}$ ). One might argue this improvement is rather limited. However, this is the result of introducing one UAV in the network. Increasing the number of UAVs will of course increase the benefit. Further investigation is required to determine if the effect will be linear or not. Nevertheless, even the earnings by introducing a single UAV is interesting for an operator, since it allows to satisfy $1 \%$ of the users that were unsatisfied by the terrestrial network for only a limited investment (about 8000 euro per drone). Furthermore, the higher the cluster cardinality, the higher the sum throughput. When increasing the cardinality

\footnotetext{
${ }^{1}$ We avoid using the notion of SINR (Signal-to-Interference-plusNoise-Ratio) as it requires the underlying assumption that interference is Gaussian, as noise. We prefer to account separately for SNR and SIR.
}

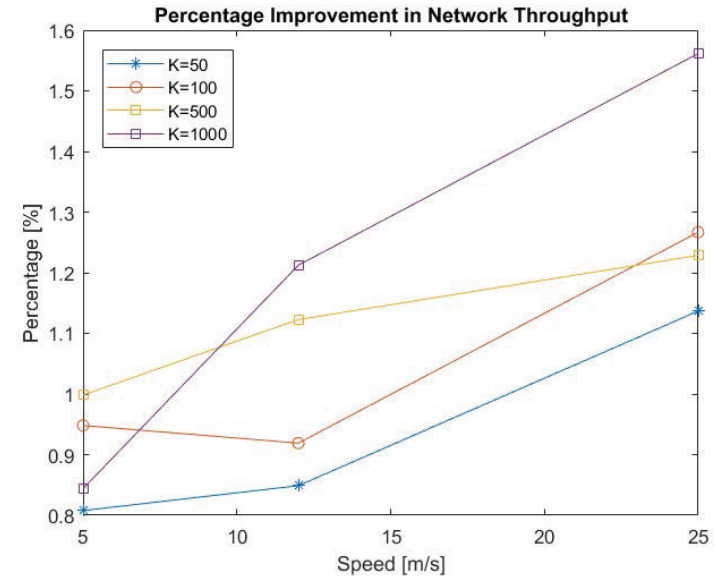

Fig. 4. Improvement percentage of sum throughput compared to the scenario without a UABS for varying UAV speed and different cluster cardinalities.

from 50 to 1000 , the sum throughput grows up to $4 \%$. When using a larger amount of clusters, the users in these clusters are closer to the centroid. Because of this, the UABS is able to cover more users when it flies over the centroid. The impact of the UAV speed on the sum throughput is limited. An improvement between $0.2 \%$ and $0.7 \%$ is noticed when the UAV accelerates from 5 to $25 \mathrm{~m} / \mathrm{s}$. When the UABS flies faster, a larger number of clusters is visited for the same simulation duration and the waiting time of each user is reduced, thus a higher success rate and sum throughput are obtained.

\section{B. Introduction of the interference management}

For the results discussed above, we neglected the fact that the UABS causes interference in the terrestrial network since it is operating in the same frequency band (Table I). In this section, we investigate the network performance when introducing the interference avoidance strategy of Section III-C. Fig. 5 shows the improvement percentage of the sum throughput compared to the scenario without a UABS for varying UABS height and speed. Note that we only consider a cluster cardinality $K$ of 1000 as the results from Section IV-A show best performance for this value. Furthermore, unless mentioned otherwise, we increased the transmit power of the UABS to $9 \mathrm{dBm}$. This increase is necessary because with a transmit power of $0 \mathrm{dBm}$, the transmit power of the UABS becomes too low with respect to the $43 \mathrm{dBm}$ transmit power of the terrestrial base station (Table I).

As one might expect, introducing an interference avoidance approach reduces the sum throughput gain obtained by using the UABS. About $0.3 \%$ of the sum throughput improvement is lost compared to when interference is not accounted for (Fig. 5 versus Fig. 4 for a height of $50 \mathrm{~m}$ ). However, we still obtain an improvement of at least $0.5 \%$ and even up to $2 \%$ when using a height of $125 \mathrm{~m}$ and a speed of $25 \mathrm{~m} / \mathrm{s}$. 


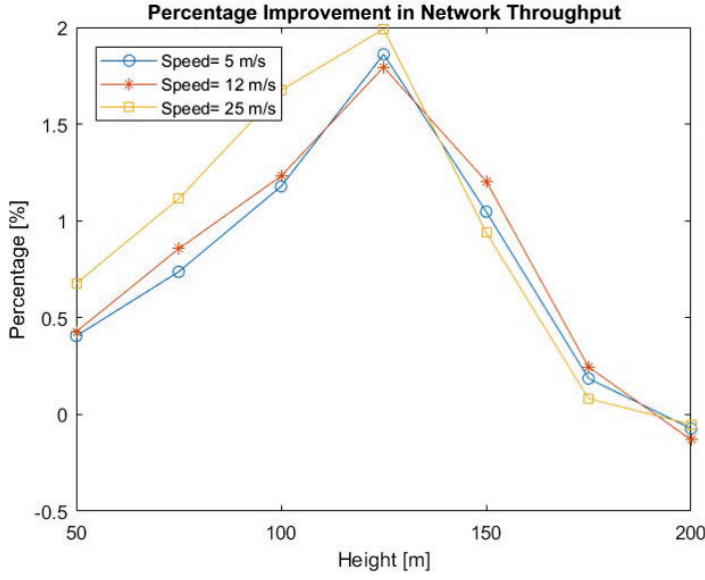

Fig. 5. Sum throughput improvement percentage compared to the scenario without a UABS for varying UABS height and speed ( $K=$ 1000).

The positive effect of the fly height on the sum throughput is nonetheless not unlimited. When flying higher than $125 \mathrm{~m}$, the sum throughput decreases again and drops even below the performance of the scenario without a UABS for a height of $200 \mathrm{~m}$. The main reason for this behaviour is the pool of available RUs that can be assigned by the UABS. When the fly height increases, the coverage range of the UABS, and thus its footprint, becomes larger. The larger this footprint, the more users are present within it and the more RUs are already assigned. Due to this the pool of the RUs available to the UABS is restrained and the UABS has not enough RUs available to serve all the unsatisfied users in the cluster. Fig. 6 shows the influence of the UABS's fly height on the amount of RUs used by TBSs inside the UABS's footprint. For a height of $150 \mathrm{~m}$, more than $1000 \mathrm{RUs}$ are already assigned, which is about half of the available RUs. For a height of $200 \mathrm{~m}$, almost all RUs are assigned by the TBSs.

Besides the fly height, also the transmit power of the UABS has an influence on its footprint. Fig. 7 show the sum throughput and the improvement percentage of this sum throughput compared to the scenario without a UABS, respectively, when varying the transmit power of the UABS for a height of $100 \mathrm{~m}$, a speed of $12 \mathrm{~m} / \mathrm{s}$ and a cluster cardinality of 1000 . Doubling the transmit power can improve the sum throughput up to $0.2 \%$ ( $9 \mathrm{dBm}$ transmit power). A higher transmit power corresponds with a larger coverage range of the UABS. Due to this the UABS can cover more unsatisfied users when visiting a cluster. Fig. 8 shows the user outage ratio due to SIR (Signal-to-Interference-Ratio) when varying the transmit power of the UABS. Increasing the transmit power from $3 \mathrm{dBm}$ to $9 \mathrm{dBm}$ results in a $11 \%$ reduction of user outage due to SIR.

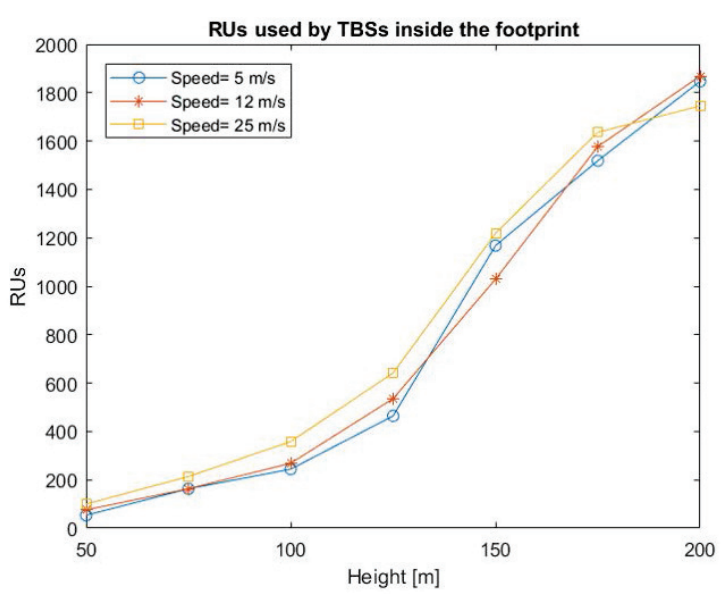

Fig. 6. Number of RUs used by TBSs inside the footprint of the UAV for varying UABS height and speed $(K=1000)$.

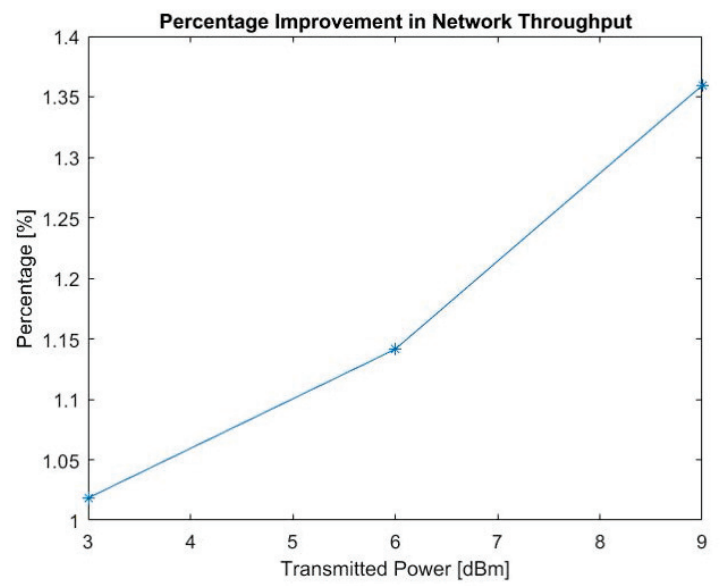

Fig. 7. Improvement percentage of the sum throughput compared to the scenario without a UABS for varying transmit powers of the UABS $(K=1000$, speed $=12 \mathrm{~m} / \mathrm{s}$, height $=100 \mathrm{~m})$.

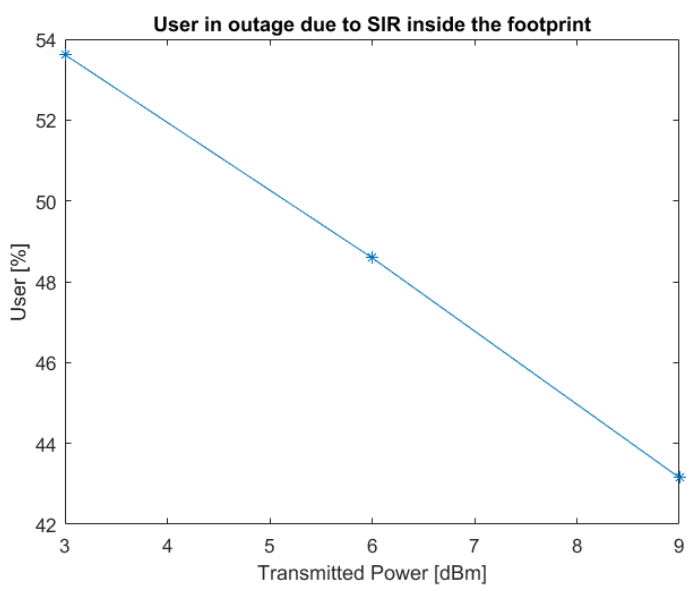

Fig. 8. User outage ratio due to SIR when varying the transmit power of the UABS $(K=1000$, speed $=12 \mathrm{~m} / \mathrm{s}$, height $=100 \mathrm{~m})$. 


\section{CONCLUSion}

This paper addresses the topic of UAV aided networks. More specifically, we consider a UABS flying over an area served by terrestrial base stations to connect those users that remained unsatisfied by the terrestrial network. A novel dynamic trajectory design for the UABS is proposed, allowing to decide on-the-go the next direction of the UABS instead of at the start of its flight time. Furthermore, we introduce an interference avoidance approach from the UABS to the terrestrial network in case they are operating in the same frequency band. These approaches are applied on an urban environment where users move and request video downloads with a minimal throughput. Using our dynamic trajectory improves the sum throughput up to almost $1.6 \%$ compared to the scenario without the UABS. The design of the trajectory is based on clusters made by nearby unsatisfied users that need to be served. Choosing a larger cluster cardinality has a positive effect on the sum even up to $4 \%$. Furthermore, it is recommended to use a speed of $25 \mathrm{~m} / \mathrm{s}$. When introducing an interference avoidance approach, one expects that the sum throughput improvement obtained by using a UABS will deteriorate. However, the decrease in sum throughput is limited to only $0.3 \%$. An improvement of up to $2 \%$ can still be obtained compared to the network without the UABS depending on the assumed cluster cardinality, and the UABS's transmit power, fly height, and speed. A fly height above $125 \mathrm{~m}$ is not eligible for the considered scenario. Future work consists of evaluating the performance of our approaches for different scenarios and introducing multiple UABSs in a single environment which will increase the sum throughput benefit.

\section{ACKNOWLEDGMENT}

M. Deruyck is a Post-Doctoral Fellow of the FWOV (Research Foundation - Flanders, Belgium). This work has been carried out within the COST CA15104 IRACON Action framework.

\section{REFERENCES}

[1] Q. Feng, J. McGeehan, E. Tameh and A. R. Nix, Path loss models for air-to-ground radio channels in urban environments, 2006 IEEE 63rd Vehicular Technology Conference, Vol. 6, pp. 29012905, 2006.

[2] A. Al-Hourani, S. Kandeepan, and S. Lardner, Optimal LAP altitude for maximum coverage, IEEE Wireless Communications Letters, Vol. 3, No. 6, pp. 569-572, 2014.

[3] A.Al-Hourani, S. Kandeepan, and A. Jamalipour, Modeling air-toground path loss for low altitude platforms in urban environments, IEEE Global Communications Conference, pp. 2898-2904, 2014.

[4] P. Ladosz, H. Oh and W. Chen, Optimal positioning of communication relay unmanned aerial vehicles in urban environments, 2016 International Conference on Unmanned Aircraft Systems (ICUAS), pp. 1140-1147, 2016.

[5] M. Mozaffari, W. Saad, M. Bennis and M. Debbah, Mobile Internet of Things: Can UAVs Provide an Energy-Efficient Mobile Architecture?, 2016 IEEE Global Communications Conference (GLOBECOM), pp. 1-6, 2016.
[6] B. Galkin, J. Kibilda and L. A. DaSilva, Deployment of UAVmounted access points according to spatial user locations in twotier cellular networks, Wireless Days (WD), pp. 1-6, 2016.

[7] V. V. C. Ravi and H. S. Dhillon, Downlink coverage probability in a finite network of unmanned aerial vehicle (UAV) base stations, Signal Processing Advances in Wireless Communications (SPAWC), 2016 IEEE 17th International Workshop on, pp. 1-5, 2016.

[8] M. Deruyck, J. Wyckmans, L. Martens, W. Joseph, Emergency AdHoc Networks by Using Drone Mounted Base Stations for a Disaster Scenario, IEEE 12th International Conference on Wireless and Mobile Computing, Networking and Communications (WiMob), pp. 1-7, 2016.

[9] V. Sharma, M. Bennis and R. Kumar, UAV-Assisted Heterogeneous Networks for Capacity Enhancement, IEEE Communications Letters, Vol. 20, No. 6, pp. 1207-1210, 2016.

[10] S. Mignardi, R. Verdone, On the Performance Improvement of a Cellular Network Supported by an Unmanned Aerial Base Station, submitted.

[11] R. Xu, D. Wunsch II, Survey of Clustering Algorithms, IEEE Transactions on Neural Networks, Vol. 16, No.3, 2005. 